\title{
Percepción del profesional contable brasileño sobre operaciones sospechosas de lavado de dinero
}

\section{Jonatas Dutra Sallaberry, Leonardo Flach}

\author{
Doutorando em Contabilidade pela Universidade Federal de Santa Catarina \\ Universidade Federal de Santa Catarina PPGC \\ Departamento de Ciências Contábeis e do Programa de Pós-Graduação em Contabilidade (PPGC) \\ Universidade Federal de Santa Catarina (UFSC)
}

Esta investigación analiza la percepción y el conocimiento de los profesionales contables brasileńos con respecto a la comunicación de los signos de lavado de dinero. El estudio se basa en la política contra el lavado de dinero de la Convención de Viena (1988), que estipula la criminalización del lavado de dinero como un medio para financiar otros delitos y distorsionar la asignación de recursos del mercado. En Brasil, el lavado de dinero fue regulado en 1998 (Ley 9.613). Actualmente, la Ley 9.613 también se aplica a los profesionales de la contabilidad y el lavado de dinero está regulado por los propios contadores (Resolución CFC 1.530, 2017). La investigación presenta un enfoque exploratorio, con un instrumento construido a partir del estándar local aplicado en forma electrónica a 215 encuestados. El análisis incluyó la aplicación de técnicas estadísticas paramétricas, y análisis descriptivos y de correlación para determinar los resultados. Estos indican que los profesionales de contabilidad se consideran responsables de actuar para combatir el lavado de dinero, pero sienten que tienen un nivel limitado de capacitación y conocimiento de las circunstancias. Además, muestran preferencia por actuar por medio de canales internos. Los principales temores que pueden afectar el comportamiento son la pérdida de empleo, las represalias y el acoso. A partir de los resultados de la percepción sobre el conocimiento de las circunstancias del lavado de dinero, las organizaciones brasileńas pueden desarrollar metodologías específicas y capacitación para mitigar los riesgos de la falta de comunicación de los empleados.

Palabras clave: blanqueo de capitales, corrupción, inteligencia financiera

\section{Perception of the Brazilian Accounting Professional on Suspected Money Laundering Operations}

This research analyzed the perception and knowledge of Brazilian accounting professionals regarding the communication of Money Laundering signs. The study is based on the Anti-Money Laundering policy of the Vienna Convention, 1988, which stipulated the criminalization of Money Laundering as a means of financing other crimes and distorting the allocation of market resources. In Brazil it was regulated in 1998 (Law 9.613, 1998), and is currently also applied to accounting professionals and regulated by the accountants themselves (CFC Resolution 1.530, 2017). The research presents an exploratory approach, with instrument built from the local standard, applied by electronic form to 215 respondents. The analysis included the application of parametric statistical techniques, descriptive and correlation analysis to determine the results. The results indicate that accounting professionals consider themselves responsible for acting to combat money laundering, but with limited level of training and knowledge of circumstances, and preference for the use of internal channels. The main fears that can affect behavior are job loss, reprisals and harassment. From the results of the perception of knowledge about Money Laundering circumstances, organizations can develop specific methodologies and training to mitigate the risks of employee noncommunication.

Keywords: Money Laundering, Corruption, Financial Intelligence 


\section{Percepção do Profissional Contábil Brasileiro sobre Operaçóes Suspeitas de Lavagem de Dinheiro}

A presente pesquisa analisou a percepção e o conhecimento de profissionais contábeis brasileiros em relação à comunicação de indícios de Lavagem de Dinheiro. O estudo fundamenta-se na política de combate à Lavagem de Dinheiro, decorrente da Convenção de Viena, 1988, que estipulou a criminalização da Lavagem de Dinheiro como meio de financiamento de outros crimes e de distorçôes na alocação dos recursos do mercado. No Brasil foi regulamentada em 1998 (Lei 9.613, 1998), sendo que atualmente também é aplicada aos profissionais contábeis e regrada pelos próprios contabilistas (Resoluçáo CFC 1.530, 2017). A pesquisa apresenta uma abordagem exploratória, com instrumento construído a partir da norma local, aplicada por formulário eletrônico a 215 entrevistados. A análise contou com a aplicação de técnicas estatísticas paramétricas, análise descritiva e de correlaçáo para apuração dos resultados. Os resultados indicam que os profissionais contábeis se consideram responsáveis por atuar em prol do combate à lavagem de dinheiro, mas com nível de capacitaçấo e conhecimento das circunstâncias em nível limitado, e preferência pelo uso de canais internos. Os principais medos que podem afetar o comportamento são a perda do emprego, represálias e perseguiçóes. A partir dos resultados da percepção sobre o conhecimento das circunstâncias de Lavagem de Dinheiro, organizaçôes podem desenvolver metodologias e treinamentos específicos para mitigar os riscos de não comunicação dos colaboradores.

Palavras-chave: Lavagem de Dinheiro, Corrupção, Inteligência Financeira

\section{Introducción}

En la última década, la sociedad brasileña ha atravesado un proceso de aparente empoderamiento para rechazar y denunciar crímenes que consumen recursos públicos y privados. Las prácticas ilícitas entre terceros transforman los recursos potenciales de inversión productiva en un instrumento para financiar las prácticas criminales más diversas (Campos \& Pereira, 2016), como la corrupción, o el tráfico de drogas y armas.

Concretamente, las apelaciones generalmente pasan por el proceso de lavado de dinero para su reintegración en el mercado. Después de ser insertado en el mercado como si su origen fuera de una actividad legal, el recurso circulará normalmente en el mercado financiero, sin dejar rastro de que proviene de un acto ilícito. Los delitos financieros involucran aspectos de importancia política, económica y moral, y representan un costo para el crecimiento y el desarrollo (Kriekebaum, 2008). Por ello, es importante de combatir el lavado de dinero.
En la década de 1980, las entidades supranacionales se dieron cuenta de que combatir el crimen implica combatir el flujo financiero empleado por las organizaciones criminales. A partir de entonces, se desarrolló una legislación para cambiar la responsabilidad de mantener la vigilancia de los actores del mercado (Coates, 2007). El profesional contable, por su experiencia en diferentes temas comerciales, posee un conocimiento relevante sobre las etapas del negocio y, en consecuencia, una mayor capacidad para diagnosticar la existencia de flujos sospechosos de recursos de lavado de dinero.

En los países desarrollados, los temas de fraude y corrupción son ampliamente debatidos por la sociedad organizada (Lehman \& Okcabol, 2005). En Brasil, este es un tema considerado solamente por los investigadores de derecho. Lo más destacado es la ampliación de la cobertura regulatoria sobre el tema (Amorim, Cardozo, \& Vicente, 2012; Gomes, Ramos, Silva, \& Santos, 2018) y el aumento de las comunicaciones (Gomes et al., 2018; Conselho de 
Controle de Atividades Financeiras [COAF], 2019), aunque en pequeños porcentajes. En un universo de casi 600.000 profesionales y organizaciones contables, ha habido poco más de 5000 comunicaciones en cinco años.

A partir de este escenario, esta investigación tiene como objetivo analizar las percepciones y el conocimiento del profesional contable sobre los signos de lavado de dinero. Se busca comprender cómo el profesional contable se percibe a sí mismo con respecto a la responsabilidad de informar transacciones sospechosas, al conocimiento de transacciones comerciales sospechosas y al nivel de capacitación, así como a la seguridad y los miedos.

Pacini, Hopwood y Sinclair (2016) justifican la importancia de esta investigación, ya que cualquier búsqueda en las noticias revela cientos de esquemas de fraude. El estudio de estos permitiría entender cómo funcionan y los factores que contribuyeron a que ocurran; así, otros esquemas de fraude podrían ser detectados y evitados (Apostolou, Apostolou, \& Thibadoux, 2015; Simon, 2012). Sin embargo, no se ha dedicado tanta atención e investigación al seguimiento de estos activos (Albrecht, Kranacher, \& Albrecht, 2008).

Comprender los resultados de la evaluación del conocimiento del profesional contable sobre el lavado de dinero puede demostrar el nivel de empoderamiento de la clase contable con respecto a la posibilidad de usar su conocimiento para la transformación social. Un entorno de mayor conocimiento sobre hechos comerciales y delitos económico-financieros puede reducir los riesgos y, en consecuencia, el costo de capital de las organizaciones (Júnior \& Moreira, 2011), debido a la mayor efectividad de la gobernanza y el control interno (Gomes et al., 2018).

\section{Referencias teórico-normativas}

El lavado de dinero probablemente es un reflejo inherente de los otros delitos con resultados financieros, ya que el procedimiento racional del criminal sería ocultar su práctica. Aunque el lavado de dinero no es un crimen nuevo, la tipificación penal y la sanción son recientes. Por ejemplo, desde los años ochenta y noventa, aparecen casos contra la criminalización, como la ley estadounidense en 1986, la ley francesa en 1987, la ley brasileńa en 1998 y la ley suiza en 2000 (Moro, 2010).

El origen del término "lavado de dinero" proviene de las lavanderías utilizadas por la mafia en la década de 1920 en los Estados Unidos, que incorporaron recursos de origen criminal en los ingresos corporativos en un intento de ganar dinero con la práctica de otros delitos (Alldridge, 2008; Martins, 2011). El mundo moderno se ha vuelto muy dinámico: ahora, son organizaciones y negocios globales los que realizan las transacciones de manera muy rápida y casi instantáneamente, pero la práctica sigue siendo la misma.

En 1988, la Convención de las Naciones Unidas contra el Tráfico Ilícito de Estupefacientes y Sustancias Psicotrópicas, también llamada "Convención de Viena", estipuló que los países signatarios penalizaran el lavado de dinero originado en el tráfico de drogas. Esto se convirtió en un hito en la lucha contra el lavado de dinero. Desde la crisis financiera internacional de 1997, los formuladores de políticas se han dado cuenta de que las distorsiones en la asignación de estos recursos pueden socavar la credibilidad y la eficiencia del sistema financiero internacional (Wechsler, 2001).

Al ocultar prácticas delictivas, los recursos utilizados o derivados de actividades ilegales no se preocupan por el desarrollo de los mercados y, por lo tanto, aceptan pérdidas anormales que no serían aceptadas en condiciones de mercado eficientes. Dichas pérdidas son 
potencialmente perjudiciales para la libre competencia, pues comprometen la entrada de nuevas empresas y el desarrollo de otros negocios. Incluso, pueden llevar a las empresas a perder competitividad y poder de negociación (Gupta, Sharan, \& Mello, 2000). No solo suponen la reducción de las ganancias de algunos emprendedores, sino también son dañinas porque, para su mantenimiento, generalmente tienen efectos variados en su entorno: el desarrollo de organizaciones criminales, corrupción, fraude, reducción de oportunidades de trabajo que estarían bajo competencia normal, aumentos de precios y debilitamiento socioeconómico de la comunidad local (Mauro, 1995). Este tipo de dinámicas ha traído riesgos para la detección y el seguimiento de los flujos financieros ilícitos; para los organismos de control; y para las propias empresas que optan por llevar a cabo sus actividades comerciales de manera legal y transparente, ya que las empresas acreditadas no quieren relacionarse a delitos (Grandis, 2011).

\subsection{Norma legal}

En Brasil, los delitos de lavado de dinero u ocultamiento de bienes, derechos y valores solo han sido penalizados por la legislación en 1998 (Ley 9.613, 1998), que tipifica el delito de "lavado" como ocultar la naturaleza, el origen, la ubicación, la disposición, el movimiento o la propiedad de bienes, o los derechos o valores derivados de diversos delitos (Presidência da República, 1998).

La mayoría de las veces, el profesional de contabilidad y finanzas realiza sus actividades laborales teniendo el recurso financiero como insumo para el desarrollo de sus tareas, tales como orientación, aplicación, inversión, registro y contabilidad; en ese sentido, no se preocupa mucho por el origen del recurso. Sin embargo, las prácticas contables pueden instrumentalizar redes corruptas o criminales, con lo que sistematizarían y permitirían su reproducción incluso sin un propósito profesional explícito (Neu, Everett, Rahaman, \& Martinez, 2013). No obstante, en el contexto penal, es importante resaltar que el financiero estaría incurriendo en la misma pena del delito de lavado. En Brasil, el que comete dicho crimen es el que 1) convierte los recursos en activos legales; 2) adquiere, recibe, intercambia, negocia, da o recibe recursos en garantía, o los mantiene, tiene en depósito, mueve o transfiere; 3) importa o exporta bienes con valores que no corresponden a los verdaderos; 4) utiliza, en actividades económicas o financieras, activos, derechos o valores derivados de delitos penales; o 5) participa en un grupo, asociación u oficina sabiendo que su actividad principal o secundaria está dirigida a la práctica de delitos (Ley 9.613, 1998, arts. 1, $\$ \$ 1$ y 2,).

La tipificación del delito de lavado de dinero ha estado acompañada de la obligación de las empresas y profesionales en segmentos específicos de informar a las agencias de control sobre la ocurrencia de transacciones sospechosas relacionadas con dicho delito. Los informes son considerados como la estrategia más efectiva para detectar irregularidades financieras (Association of Certified Fraud Examiners [ACFE], 2018). Inicialmente, por la Ley de 1998 (Ley 9.613, 1998, art. 9), las personas jurídicas, cuya actividad consiste en operar en el mercado financiero y sus productos, tienen la responsabilidad de informar a los organismos de control cualquier actividad sospechosa de blanqueo de capitales. Posteriormente, estas obligaciones se extendieron a individuos en diversos campos.

Las enmiendas a la Ley de Lavado en 2012 (Ley 12.683, artículo 9, XIV) incluyeron en el alcance de las normas otros bienes y servicios potencialmente empleados en eventos de lavado de dinero, así como algunas actividades profesionales de naturaleza técnica 
y producto subjetivo, como "proporcionar servicios de asesoramiento, consultoría, contabilidad, auditoría, asesoramiento o asistencia de cualquier tipo en transacciones para":

1) la compra y venta de bienes inmuebles, establecimientos comerciales o industriales o intereses patrimoniales de cualquier tipo;

2) gestión de fondos, valores u otros activos;

3) la creación o administración de cuentas bancarias, de inversión o de valores;

4) la creación, operación o gestión de empresas de cualquier tipo, fundaciones, fondos fiduciarios o estructuras similares;

5) financiero, corporativo o inmobiliario; $y$

6) venta o adquisición de derechos de contratos relacionados con deportes profesionales o actividades artísticas [traducción propia] (Presidência da República, 1998; 2012).

Este conjunto de servicios de asesoramiento, consultoría, contabilidad, auditoría, asesoramiento u operaciones de asistencia normalmente se ajusta a las actividades realizadas por los profesionales de contabilidad y finanzas. Todas las empresas y profesionales relacionados deben informar a las Unidad de Inteligencia Financiera (en adelante, UIF), nombrado "Consejo de Control de Actividades Financieras" (en adelante, COAF) en Brasil.

El COAF también fue creado en la Ley de Lavado de Activos (Ley 9.613, 1998) con el fin de disciplinar; hacer cumplir las sanciones administrativas; y recibir, examinar e identificar casos sospechosos de actividades ilegales por medio de la acumulación de la actividad de regulación para segmentos, para los cuales no existe un organismo supervisor o regulador adecuado en el país (Presidência da República, 1998).

\subsection{Reglamento del Consejo Nacional de Contabilidad}

En el caso de los profesionales contables que actúan en el ámbito de la profesión reglada (Decreto-Ley 9.295/1946), las reglas e indicaciones de transacciones sospechosas están explícitas en el estándar profesional 2017 (Resolución CFC 1.530/2017, que reemplazó a la Resolución CFC 1.445/2013), que aborda los procedimientos que deben seguir los profesionales y organizaciones contables para cumplir con las obligaciones establecidas en la Ley de Lavado de Activos. El estándar, que es bastante completo, trata de regular y abordar los puntos previstos en la Ley de Lavado. Entre ellos, están incluidos el alcance del estándar, que indica quiénes serían los profesionales a los que se llega con la obligación; las pautas de la política de prevención y análisis de riesgos; los requisitos de los registros de los clientes; y los registros de operaciones, almacenamiento y preservación de información, así como las comunicaciones obligatorias y sospechosas.

Con respecto a las comunicaciones, hay dos tipos de informes de operaciones a la UIF: obligatorios/automáticos y sospechosos. Las comunicaciones automáticas u obligatorias son las que necesariamente deben comunicarse a la UIF porque han alcanzado un cierto valor umbral, independientemente de cualquier análisis de mérito o sospecha. En el caso de los profesionales contables, la Resolución 1.530 (2017, artículo 6) establece la comunicación obligatoria en montos, aunque momentáneamente fraccionados: a) adquisición de activos y pagos a terceros, en efectivo, por más de BRL 50.000,00 (cerca de USD 12.500) por operación; y/o b) incorporación y/o aumento de capital 
con pago, en especie, de más de BRL 100.000,00 (cerca de USD 25.000) en un solo mes calendario.

Las comunicaciones sospechosas son aquellas que, debido a sus características, deben ser analizadas cuidadosamente y están previstas en la norma profesional (Resolución CFC 1.530, 2017, artículo 5). Deben indicar los siguientes puntos:

i. una operación que no parece ser el resultado de las actividades habituales del cliente o su negocio;

ii. una operación cuyo origen o base económica o jurídica no es claramente verificable;

iii. una operación incompatible con el patrimonio, la capacidad económica financiera, la actividad o la línea de negocio del cliente;

iv. una operación con un cliente cuyo beneficiario final no puede ser identificado;

v. una operación o propuesta que involucra una entidad legal domiciliada en jurisdicciones consideradas por el Grupo de Acción de Alto Riesgo de Lavado de Dinero y Financiamiento del Terrorismo (en adelante, Gafi); o con deficiencias en la prevención y lucha contra el lavado de dinero y el financiamiento del terrorismo, o países considerados por tributación favorecida y/o régimen tributario privilegiado;

vi. una operación o propuesta que involucra a una entidad legal cuyos beneficiarios finales, socios, accionistas, abogados o representantes legales están domiciliados en jurisdicciones consideradas por el Gafi como de alto riesgo, o con deficiencias estratégicas en la prevención y lucha contra el lavado de dinero y el financiamiento del terrorismo; o países o dependencias consideradas por la Receita Federal do Brasil (en adelante, RFB) sobre impuestos favorecidos y/o régimen fiscal privilegiado;

vii. una operación injustificadamente compleja o con costos más altos destinados a dificultar el seguimiento de los recursos o la identificación del propósito real de la operación;

viii. una operación destinada a alterar o manipular las características de las operaciones financieras o identificar el propósito real de la operación;

ix. una operación aparentemente ficticia o evidencia de sobreprecio o subprecio;

x. una operación con cláusulas que establecen condiciones incompatibles con las practicadas en el mercado;

xi. cualquier intento de fraccionar valores para evitar la comunicación en especie; y

xii. cualquier otra operación que, considerando las partes y otras personas involucradas, los montos, la forma de pago y los medios de pago, o la falta de base económica o legal, puedan constituir serias indicaciones de la ocurrencia de los delitos previstos en la Ley de Lavado de Dinero.

Estas comunicaciones enviadas a la UIF se analizan más a fondo y se pueden enviar a las autoridades competentes (policía, fiscales, agencias de tesorería) cuando se atribuye al establecimiento la posibilidad de delitos, pruebas fundadas de su práctica o cualquier otro acto ilícito procedimientos de investigación. La notificación obligatoria o sospechosa a la UIF es solo una de las obligaciones establecidas por la Ley de Lavado de Dinero, que requiere que las entidades alcanzadas establezcan registros actualizados de clientes (artículo 10, I), registros de transacciones (II), controles internos (III) y custodia ( $(2)$, elementos típicos de las 
actividades, y rutinas de contabilidad. Estos aspectos llevan la contabilidad al corazón de las actividades de monitoreo y reporte de cualquier evidencia de delitos de lavado de dinero.

Incluso con las nuevas regulaciones, y la aparición de varias investigaciones y operaciones policiales con amplia divulgación en los medios, los informes de operaciones sospechosas de lavado de dinero han sido escasos en los diversos segmentos no bancarios, según lo informado por Gomes et al. (2018). En otros países, también se han establecido nuevos mecanismos que aún requieren más estudio (Coates, 2007). Por otro lado, según las estadísticas de la UIF (COAF, 2019), el escenario entre los profesionales de la contabilidad presenta un nivel muy bajo de comunicaciones. En poco más de cuatro años de las resoluciones del Consejo Nacional de Contabilidad, los 517000 profesionales de contabilidad y 68000 organizaciones de contabilidad hicieron solo 1875 informes de transacciones sospechosas de sus clientes, y un total de 5531 comunicaciones en especie ya incluidas. (Conselho Federal de Contabilidade [CFC], 2019)

Tabla 1. Estadística de informes

\begin{tabular}{|l|c|c|c|c|c|c|}
\hline \multicolumn{1}{|c|}{ Informes } & 2014 & 2015 & 2016 & 2017 & 2018 & Monto \\
\hline $\begin{array}{l}\text { Operaciones } \\
\text { obligatorias } \\
\text { en efectivo }\end{array}$ & 60 & 1163 & 719 & 763 & 952 & 3657 \\
\hline $\begin{array}{l}\text { Operaciones } \\
\text { sospechosas }\end{array}$ & 44 & 245 & 496 & 594 & 496 & 1875 \\
\hline $\begin{array}{l}\text { Suma de } \\
\text { informes }\end{array}$ & 104 & 1408 & 1215 & 1356 & 1448 & 5531 \\
\hline
\end{tabular}

Fuente: COAF (2019) y CFC (2019).

La baja tasa de informes de transacciones sospechosas puede deberse a incentivos monetarios o de comportamiento, a falta de conocimiento sobre la ley y transacciones sospechosas, y a los diversos negocios. Dadas las recientes regulaciones y la ausencia de comunica- ciones, se considera la necesidad de conocer la percepción del profesional contable sobre su responsabilidad, su conocimiento y los medios de ejecución de estos informes. Los profesionales que están dentro del alcance de la norma también deben declarar anualmente si no han hecho ninguna comunicación. Con respecto a los contadores que informan no ocurrencia, los profesionales comunicadores de no ocurrencia han representado aproximadamente al $25 \%$ de los profesionales registrados en los consejos de contabilidad.

\section{Método de investigación}

La presente investigación presenta un enfoque exploratorio del problema. Se han aplicado técnicas estadísticas paramétricas para determinar los resultados, así como un análisis descriptivo de los objetivos. El instrumento de investigación fue construido a partir de las preguntas que contienen los términos de los reglamentos de riesgos de lavado de dinero (Resolución CFC 1.530, 2017), que establecen la obligación de los profesionales de la contabilidad de comunicar ciertos hechos a los organismos de control, principalmente, derivados de la Ley de Lavado de Dinero (COAF, 2017). Las posibles repuestas se plantearon en escalas Likert de 10 puntos, lo que permite una mayor distancia entre las categorías de ellas. Los extremos se marcaron como 1 ("ignorancia total") y 10 ("conocimiento total”). Además, se incluyeron temas discutidos con un grupo de diez especialistas en contabilidad con experiencia en control interno y externo, compliance, e investigación para capturar la percepción del individuo de su grado de responsabilidad, conocimiento y capacidad para informar de forma abierta cuestiones de control, como la capacitación y el desempeńo profesional. Las preguntas del instrumento se muestran en la tabla 2. El cuestionario fue aplicado originalmente en portugués.

Contabilidad y Negocios (16) 31, 2021 / e-ISSN 2221-724X 
Tabla 2. Cuestionario de investigación

\section{CUESTIONARIO}

1) ¿ Se considera un profesional responsable de actuar para combatir el lavado de dinero y los delitos relacionados?

2) Según su conocimiento sobre la conducta contra el lavado de dinero, podría reconocer circunstancias o hechos que indiquen:

2.a) ¿̨una operación que aparentemente no resulta de las actividades habituales de un cliente o su negocio?

2.b) ¿una operación cuyo origen o base económica o legal no es claramente identificable?

2.c) ¿̨una operación incompatible con el patrimonio del cliente, capacidad económica y financiera, o actividad o negocio?

2.d) ¿̨una operación con un cliente cuyo beneficiario final no puede ser identificado?

2.e) ¿̨una operación o propuesta que involucre a una entidad legal domiciliada en jurisdicciones consideradas por el Gafi, como de alto riesgo o con deficiencias en la prevención y lucha contra el lavado de dinero y el financiamiento del terrorismo, o países o dependencias consideradas por el servicio de impuestos de tributación favorecida y/o régimen tributario privilegiado?

2.f) ¿una operación o propuesta que involucre a una entidad legal cuyos beneficiarios finales, socios, accionistas, abogados o representantes legales estén domiciliados en jurisdicciones consideradas por Gafi como de alto riesgo, o con deficiencias estratégicas en la prevención y lucha contra el lavado de dinero y el financiamiento del terrorismo, o países o dependencias consideradas por la RFB para favorecer la tributación y / o el régimen fiscal privilegiado?

2.g) ¿una operación injustificadamente compleja, o con costos más altos para dificultar el seguimiento de los recursos o identificar el verdadero propósito de la operación?

2.h) ¿ una operación con características destinadas a alterar o manipular las operaciones financieras, o la identidad del propósito real de la operación?

2.i) ¿una operación aparentemente ficticia, o la evidencia de sobreprecio o subprecio?

2.j) ¿una operación con cláusulas que establecen condiciones incompatibles con las practicadas en el mercado?

2.k) ¿algún intento de fraccionar valores para evitar alcanzar valores que requieren comunicación automática?

2.1) ¿cualquier otra operación que, considerando las partes y otras personas involucradas, los montos, la forma de pago y los medios de pago, o la falta de base económica o legal, puedan constituir serias indicaciones de la ocurrencia de los delitos previstos en la Ley 9.613/1998 o se refieran a ellos?

2.m) ¿̨la adquisición de activos y pagos a terceros en efectivo por más de BRL 50.000,00 por operación?

2.n) ¿̇la incorporación de capital a la empresa y/o aumento de capital con pago en especie de más de BRL 100.000,00 en un solo mes calendario?

3) ¿¿Ha estudiado la Resolución CFC 1.530/2017 y 1.445/2013, que se ocupan de los procedimientos que deben seguir los profesionales y organizaciones contables para cumplir con las obligaciones establecidas en la Ley de Lavado de Activos (Ley. 9.613, 1998)?

4) ¿¿Se considera calificado para actuar de acuerdo con la Resolución CFC 1.530/2017?

5) ¿`Se califica como un profesional de contabilidad que debe informar a COAF (profesionales y organizaciones de contabilidad que brindan, incluso si los hay, servicios de asesoramiento, consultoría, contabilidad, auditoría, asesoramiento o asistencia de cualquier tipo en las siguientes operaciones, realizadas por personas físicas o jurídicas: 1) compra y venta de bienes inmuebles, establecimientos comerciales o industriales, o intereses patrimoniales de cualquier naturaleza; 2) gestión de fondos, valores u otros activos; 3 ) apertura o gestión de cuentas bancarias, ahorro, inversión o valores; 4) creación, operación o administración de compañías de cualquier tipo, y fundaciones, fondos fiduciarios o estructuras similares; 5) financiero, corporativo o inmobiliario; y 6) venta o adquisición de derechos sobre contratos; relacionado con deportes profesionales o actividades artísticas)? 


\section{CUESTIONARIO}

6) ¿¿Ha sido supervisado alguna vez de manera externa por el desempeño correcto con respecto a la comunicación de operaciones sospechosas a las agencias de control?

7) ¿Qué enlace profesional lo caracteriza mejor?

8) ¿Cuál es su grado más alto completado?

9) ¿Ha oído hablar de NOCLAR (Responding to Non-Compliance with Laws and Regulations)?

10) Si conociera la evidencia de un acto ilegal, ¿informaría a su empresa internamente?

11) Si fuera consciente de la evidencia de un acto ilegal, ¿se aseguraría de informar fuera de su empresa al organismo de control?

12) Si ha estado al tanto de la evidencia de un acto ilegal, ¿cuál ha sido su mayor temor al reportar un hecho que ocurrió en su empresa? Fuente: Resolución CFC 1.530/2017.

El cuestionario fue construido usando el método de "encuesta" en la herramienta en línea de Google Forms (https://goo.gl/forms/FHrRpmrO6PowRw7J3) y se refirió a profesionales de negocios enfocados en contabilidad. En total, se recopilaron 215 respuestas, que alcanzaron un Alfa de Cronbach de 0,9278 (Correlación promedio: 0,3687, escala de ítems: 22); se procesaron en una hoja de cálculo y se analizaron estadísticamente en el Software Stata 13, lo que garantiza la confiabilidad del instrumento (Cronbach, 1951).

\section{Análisis de datos}

La estadística descriptiva busca describir y evaluar un grupo en particular sin proponer conclusiones o inferencias sobre un grupo más grande (Fávero, Belfiore, Takamatsu, \& Suzart, 2014). Un análisis descriptivo permite conocer la muestra, y las circunstancias y características de los individuos. Estos aspectos pueden ayudar en la interpretación de los resultados. En este contexto, las características se evaluaron discrecionalmente desde el punto de vista de las variables de control, que representan características notoriamente relacionadas con la retención de conocimiento: capacitación y experiencia profesional (actua- ción). El alcance de la investigación apunta a tener una mejor evaluación de los profesionales de contabilidad. Sin embargo, no se descartaron las respuestas de profesionales de otras áreas, ya que el desempeño del contador a menudo se confunde con actividades en el campo de negocios relacionadas a la atribución de finanzas, logística, y recursos humanos, entre otras áreas de especialización contable.

La mayoría de los encuestados tienen una licenciatura o posgrado con una monografía relacionada a la contabilidad. Respecto de la actuación, la muestra tiene mayor diversidad: predominan los empleados de organizaciones contables $(30,1 \%)$ por encima de los de organizaciones no contables $(18,2 \%)$, aunque en actividades relacionadas con la contabilidad. $\mathrm{Al}$ ańadir los encuestados autónomos en actividades contables, el porcentaje de los profesionales con experiencia laboral en rutinas contables llega a 60,7\%; además, 25.6\% de los encuestados manifestaron tener al menos una profundización teórica en contabilidad. A partir de las respuestas de estos profesionales con capacitación y desempeño predominantemente contable, es posible asegurar una fidelidad relativa de los datos con el escenario local. 
Tabla 3. Descripción de formación y actuación

\begin{tabular}{|c|c|c|c|c|c|c|c|c|c|c|c|c|c|c|c|c|}
\hline \multirow[t]{2}{*}{ Formación } & \multirow[t]{2}{*}{$\%$} & \multicolumn{3}{|c|}{$\begin{array}{l}\text { Q3d } \\
\text { Estudio de } \\
\text { legislación }\end{array}$} & \multicolumn{3}{|c|}{$\begin{array}{l}\text { Q4d Res. } \\
\text { CFC.1.530 }\end{array}$} & \multicolumn{3}{|c|}{$\begin{array}{c}\text { Q5d } \\
\text { Obligación }\end{array}$} & \multicolumn{3}{|c|}{$\begin{array}{c}\text { Q6d } \\
\text { Inspección }\end{array}$} & \multicolumn{3}{|c|}{$\begin{array}{c}\text { Q9d } \\
\text { NOCLAR }\end{array}$} \\
\hline & & N: 0 & S: 1 & $\mathrm{~N}$ & $\mathrm{~N}: 0$ & S: 1 & $\mathrm{~N}$ & $\mathrm{~N}: 0$ & S: 1 & $\mathrm{~N}$ & $\mathrm{~N}: 0$ & S: 1 & $\mathrm{~N}$ & $\mathrm{~N}: 0$ & S: 1 & $\mathrm{~N}$ \\
\hline Doctor & 0,02 & 1 & 2 & 3 & 1 & 2 & 3 & 1 & 2 & 3 & 1 & 2 & 3 & 0 & 2 & 2 \\
\hline Maestro & 0,06 & 5 & 6 & 11 & 6 & 5 & 11 & 5 & 6 & 11 & 8 & 3 & 11 & 0 & 3 & 3 \\
\hline Especialista & 0,13 & 4 & 18 & 22 & 7 & 15 & 22 & 11 & 11 & 22 & 19 & 3 & 22 & 8 & 5 & 13 \\
\hline Licenciado en Contabilidad & 0,64 & 37 & 75 & 112 & 38 & 74 & 112 & 40 & 71 & 111 & 82 & 28 & 110 & 58 & 28 & 86 \\
\hline Técnico contable & 0,16 & 14 & 14 & 28 & 15 & 13 & 28 & 12 & 16 & 28 & 24 & 4 & 28 & 16 & 9 & 25 \\
\hline \multirow{2}{*}{ Suma } & \multirow{2}{*}{100} & 61 & 115 & 176 & 67 & 109 & 176 & 69 & 106 & 175 & 134 & 40 & 174 & 82 & 47 & 129 \\
\hline & & 0,35 & 0,65 & 1,00 & 0,38 & 0,62 & 1,00 & 0,39 & 0,61 & 1,00 & 0,77 & 0,23 & 1,00 & 0,64 & 0,36 & 1,00 \\
\hline Actividad & $\%$ & $\mathrm{~N}: 0$ & S: 1 & $\mathrm{~N}$ & N: 0 & S: 1 & $\mathrm{~N}$ & N: 0 & S: 1 & $\mathrm{~N}$ & N: 0 & S: 1 & $\mathrm{~N}$ & $\mathrm{~N}: 0$ & S: 1 & $\mathrm{~N}$ \\
\hline Academia & 25,6 & 24 & 30 & 54 & 26 & 28 & 54 & 21 & 33 & 54 & 46 & 8 & 54 & 26 & 14 & 40 \\
\hline Organización contable & 30,1 & 19 & 44 & 63 & 18 & 45 & 63 & 20 & 43 & 63 & 45 & 17 & 62 & 34 & 21 & 55 \\
\hline Empleado contable & 18,2 & 14 & 24 & 38 & 16 & 22 & 38 & 19 & 19 & 38 & 30 & 8 & 38 & 17 & 7 & 24 \\
\hline $\begin{array}{l}\text { Otras organizaciones y } \\
\text { actividades }\end{array}$ & 13,4 & 17 & 11 & 28 & 18 & 10 & 28 & 23 & 5 & 28 & 24 & 4 & 28 & 17 & 4 & 21 \\
\hline $\begin{array}{l}\text { Autónomo en asesora- } \\
\text { miento y consultoría }\end{array}$ & 8,6 & 7 & 11 & 18 & 5 & 13 & 18 & 4 & 13 & 17 & 10 & 7 & 17 & 14 & 1 & 15 \\
\hline $\begin{array}{l}\text { Autónomo em auditoría y } \\
\text { pericial }\end{array}$ & 3,8 & 2 & 6 & 8 & 3 & 5 & 8 & 4 & 4 & 8 & 7 & 1 & 8 & 1 & 3 & 4 \\
\hline \multirow{2}{*}{ Suma } & \multirow{2}{*}{100} & 83 & 126 & 209 & 86 & 123 & 209 & 91 & 117 & 208 & 162 & 45 & 207 & 109 & 50 & 159 \\
\hline & & 0,40 & 0,60 & 1,00 & 0,41 & 0,59 & 1,00 & 0,44 & 0,56 & 1,00 & 0,78 & 0,22 & 1,00 & 0,69 & 0,31 & 1,00 \\
\hline
\end{tabular}

Fuente: Elaboración propia a partir de los análisis estadísticos.

Los resultados tabulados de las respuestas nos permiten identificar que solo $60 \%$ de los encuestados estudiaron los estándares de prevención y lucha contra el lavado de dinero establecidos por el organismo profesional de clase (Q3d), mientras que 59\% de los participantes se consideran capaces de cumplir con los requisitos de los estándares de lavado de dinero (Q4d). En relación a una perspectiva complementaria de la actualización del conocimiento, en la pregunta sobre el conocimiento de la existencia de NOCLAR (Responding to Non-Compliance with Laws and Regulations), solo el 31\% indicó conocer su existencia (Q9d).
La percepción de control también es baja: $56 \%$ de los entrevistados indican que están dentro del alcance del estándar que requiere que el profesional contable informe el lavado de dinero (Q5d), mientras que 22\% de los participantes indicaron que ya habían sido supervisados ante posibles comunicaciones de operaciones sospechosas (Q6d). Luego del proceso de construcción de objetivos, en el análisis de los resultados de la respuesta, los profesionales informan un conocimiento promedio de 6,95 de las circunstancias o hechos que indican sospecha de lavado de dinero. Lo más destacado del alto conocimiento consiste en las afirmaciones Q2A 
(aparentemente, no es el resultado de las actividades habituales de un cliente o su negocio) y Q2C (operación incompatible con los activos, la capacidad económica financiera, la actividad o el negocio del cliente), con niveles de reconocimiento de 7,44 y 7,68 respectivamente. Estas serían las hipótesis en las que el profesional de negocios sería más capaz de percibir el hecho. Por otro lado, las afirmaciones Q2e (operación o propuesta que involucra a una entidad legal domiciliada en jurisdicciones consideradas por el Gafi, o con deficiencias en la prevención y el combate del lavado de dinero y la financiación del terrorismo) y Q2f (operación o propuesta que involucra a una entidad legal cuyos beneficiarios finales, socios, accionistas, abogados o representantes legales están domiciliados en jurisdicciones consideradas por Gafi como de alto riesgo, o con deficiencias estratégicas para prevenir y combatir el lavado de dinero y el financiamiento del terrorismo) serían las hipótesis en las que el profesional es menos capaz de percibir el hecho, con grados de 5,92 y 5,95, respectivamente.

La principal deficiencia en Q2e es el movimiento relacionado con paraísos fiscales conocidos que pueden ayudar en el lavado relacionado con el financiamiento del terrorismo. En general, la UIF nacional divulga la lista de jurisdicciones (países) consideradas de alto riesgo. La cuestión Q2f señala la dificultad de conocer a los beneficiarios y las cuentas relacionadas en el extranjero. La brecha de conocimiento que falta es relevante, pues es mayor al 40\%. Aun así, estos son valores que indican que el profesional tiene una mayor capacidad para percibir el lavado de dinero que no darse cuenta de este hecho. La principal preocupación proviene de los resultados de la desviación estándar (SD) de las afirmaciones que representan la mayor variabilidad en el universo, pues alcanzan valores SD de casi 2,5 , correspondiente a $25 \%$ de la puntuación. Estos resultados indican una brecha de oportunidad para la capacitación. En promedio, el conocimiento percibido se evaluó en 6,95 , en un universo del
1 al 10, con los promedios más altos y más bajos indicados previamente, como se muestra en la Tabla 4.

Tabla 4. Estadística de reconocimiento de evidencia

\begin{tabular}{|c|c|c|c|c|c|}
\hline Cuestión & N & PROMED & SD & MIN & MAX \\
\hline Q2A & 211 & 7,436019 & 1,966246 & 1 & 10 \\
\hline Q2B & 211 & 7,161137 & 1,89551 & 2 & 10 \\
\hline Q2C & 213 & 7,676056 & 2,081645 & 1 & 10 \\
\hline Q2D & 213 & 7,258216 & 2,133292 & 1 & 10 \\
\hline Q2E & 213 & 5,924883 & 2,497922 & 1 & 10 \\
\hline Q2F & 212 & 5,95283 & 2,406083 & 1 & 10 \\
\hline Q2G & 213 & 6,666667 & 2,237474 & 1 & 10 \\
\hline Q2H & 212 & 6,886792 & 2,197891 & 1 & 10 \\
\hline Q2I & 213 & 7,239437 & 2,174887 & 1 & 10 \\
\hline Q2J & 212 & 7,146226 & 2,279589 & 1 & 10 \\
\hline Q2K & 213 & 6,962441 & 2,184529 & 1 & 10 \\
\hline Q2L & 212 & 6,891509 & 2,072735 & 1 & 10 \\
\hline Q2M & 213 & 7,023474 & 2,366116 & 1 & 10 \\
\hline Q2N & 213 & 7,15493 & 2,379043 & 1 & 10 \\
\hline Promedio & 213 & 6,951641 & 1,705009 & 1,857143 & 10 \\
\hline
\end{tabular}

Fuente: Elaboración propia a partir de los análisis estadísticos.

El siguiente procedimiento consiste en el análisis de correlación de los indicadores. La correlación es la medida estandarizada de la relación entre dos variables (con fuerza y dirección), siempre entre los rangos de $0 \mathrm{a}$ 1. Una correlación cercana a cero indica que las dos variables no están relacionadas; mientras tanto, la más cercana a 1 indica una relación alta entre las variables, y que sería posible establecer una relación lineal entre las variables debido a la alta correlación o entre algunos grupos de variables. Las variables se pueden segregar en distintos grupos: el primer compuesto del locus de responsabilidad (Q1d), el segundo grupo de percepción del conocimiento sobre los hechos indicados (Q2a, Q2b, Q2c, Q2d, Q2e, Q2f, Q2g, Q2h, Q2i, Q2j, Q2k, Q21, Q2m, Q2n), el nivel de habilidad y conocimiento (Q3d, Q4d, Q6d, Q9d) y la propensión a informar (Q10d, Q11d). 
Tabla 5. Correlación de variables

\begin{tabular}{|c|c|c|c|c|c|c|c|c|c|c|c|c|c|c|c|c|c|c|c|c|c|c|}
\hline & Q1d & Q2A & Q2B & Q2C & Q2D & Q2E & Q2F & Q2G & Q2H & Q2I & Q2J & Q2K & Q2L & Q2M & Q2N & Q3d & Q4d & Q5d & Q6d & Q9d & Q10d & Q11d \\
\hline Q1d & 1,0000 & & & & & & & & & & & & & & & & & & & & & \\
\hline \multirow[t]{2}{*}{ Q2A } & 0,1673 & 1,0000 & & & & & & & & & & & & & & & & & & & & \\
\hline & 0,0160 & & & & & & & & & & & & & & & & & & & & & \\
\hline \multirow[t]{2}{*}{ Q2B } & 0,1998 & 0,6863 & 1,0000 & & & & & & & & & & & & & & & & & & & \\
\hline & 0,0039 & 0,0000 & & & & & & & & & & & & & & & & & & & & \\
\hline \multirow[t]{2}{*}{ Q2C } & 0,1724 & 0,6513 & 0,6470 & 1,0000 & & & & & & & & & & & & & & & & & & \\
\hline & 0,0130 & 0,0000 & 0,0000 & & & & & & & & & & & & & & & & & & & \\
\hline \multirow[t]{2}{*}{ Q2D } & 0,2564 & 0,5335 & 0,5573 & 0,5139 & 1,0000 & & & & & & & & & & & & & & & & & \\
\hline & 0,0002 & 0,0000 & 0,0000 & 0,0000 & & & & & & & & & & & & & & & & & & \\
\hline \multirow[t]{2}{*}{ Q2E } & 0,2293 & 0,3569 & 0,4922 & 0,3436 & 0,5047 & 1,0000 & & & & & & & & & & & & & & & & \\
\hline & 0,0009 & 0,0000 & 0,0000 & 0,0000 & 0,0000 & & & & & & & & & & & & & & & & & \\
\hline \multirow[t]{2}{*}{ Q2F } & 0,2051 & 0,3573 & 0,4609 & 0,2877 & 0,5070 & 0,7960 & 1,0000 & & & & & & & & & & & & & & & \\
\hline & 0,0030 & 0,0000 & 0,0000 & 0,0000 & 0,0000 & 0,0000 & & & & & & & & & & & & & & & & \\
\hline \multirow[t]{2}{*}{ Q2G } & 0,3244 & 0,4952 & 0,6346 & 0,4709 & 0,5952 & 0,6319 & 0,6958 & 1,0000 & & & & & & & & & & & & & & \\
\hline & 0,0000 & 0,0000 & 0,0000 & 0,0000 & 0,0000 & 0,0000 & 0,0000 & & & & & & & & & & & & & & & \\
\hline \multirow[t]{2}{*}{ Q2H } & 0,1984 & 0,5274 & 0,5473 & 0,5375 & 0,4975 & 0,5842 & 0,5752 & 0,6804 & 1,0000 & & & & & & & & & & & & & \\
\hline & 0,0042 & 0,0000 & 0,0000 & 0,0000 & 0,0000 & 0,0000 & 0,0000 & 0,0000 & & & & & & & & & & & & & & \\
\hline \multirow[t]{2}{*}{ Q2I } & 0,1637 & 0,5543 & 0,5169 & 0,5350 & 0,5417 & 0,5139 & 0,4622 & 0,6465 & 0,7396 & 1,0000 & & & & & & & & & & & & \\
\hline & 0,0184 & 0,0000 & 0,0000 & 0,0000 & 0,0000 & 0,0000 & 0,0000 & 0,0000 & 0,0000 & & & & & & & & & & & & & \\
\hline \multirow[t]{2}{*}{ Q2J } & 0,1268 & 0,4634 & 0,4827 & 0,4938 & 0,5285 & 0,5281 & 0,5361 & 0,6594 & 0,7007 & 0,7393 & 1,0000 & & & & & & & & & & & \\
\hline & 0,0694 & 0,0000 & 0,0000 & 0,0000 & 0,0000 & 0,0000 & 0,0000 & 0,0000 & 0,0000 & 0,0000 & & & & & & & & & & & & \\
\hline \multirow[t]{2}{*}{ Q2K } & 0,1340 & 0,4669 & 0,5367 & 0,5450 & 0,4809 & 0,5657 & 0,5468 & 0,6594 & 0,6457 & 0,6502 & 0,7264 & 1,0000 & & & & & & & & & & \\
\hline & 0,0542 & 0,0000 & 0,0000 & 0,0000 & 0,0000 & 0,0000 & 0,0000 & 0,0000 & 0,0000 & 0,0000 & 0,0000 & & & & & & & & & & & \\
\hline \multirow[t]{2}{*}{ Q2L } & 0,2224 & 0,5106 & 0,5513 & 0,5467 & 0,5792 & 0,5879 & 0,5981 & 0,6791 & 0,7532 & 0,7118 & 0,7092 & 0,7397 & 1,0000 & & & & & & & & & \\
\hline & 0,0013 & 0,0000 & 0,0000 & 0,0000 & 0,0000 & 0,0000 & 0,0000 & 0,0000 & 0,0000 & 0,0000 & 0,0000 & 0,0000 & & & & & & & & & & \\
\hline \multirow[t]{2}{*}{ Q2M } & 0,2517 & 0,3836 & 0,4688 & 0,5311 & 0,4455 & 0,5454 & 0,4984 & 0,5797 & 0,6288 & 0,5773 & 0,5848 & 0,6508 & 0,6583 & 1,0000 & & & & & & & & \\
\hline & 0,0003 & 0,0000 & 0,0000 & 0,0000 & 0,0000 & 0,0000 & 0,0000 & 0,0000 & 0,0000 & 0,0000 & 0,0000 & 0,0000 & 0,0000 & & & & & & & & & \\
\hline \multirow[t]{2}{*}{ Q2N } & 0,1522 & 0,4616 & 0,4721 & 0,5055 & 0,4661 & 0,4552 & 0,4735 & 0,5636 & 0,5886 & 0,6410 & 0,5706 & 0,5874 & 0,6958 & 0,7284 & 1,0000 & & & & & & & \\
\hline & 0,0286 & 0,0000 & 0,0000 & 0,0000 & 0,0000 & 0,0000 & 0,0000 & 0,0000 & 0,0000 & 0,0000 & 0,0000 & 0,0000 & 0,0000 & 0,0000 & & & & & & & & \\
\hline \multirow{2}{*}{ Q3d } & 0,3716 & 0,1629 & 0,2437 & 0,2107 & 0,2265 & 0,2701 & 0,3032 & 0,3295 & 0,2631 & 0,2634 & 0,2223 & 0,1917 & 0,3097 & 0,2106 & 0,2595 & 1,0000 & & & & & & \\
\hline & 0,0000 & 0,0179 & 0,0004 & 0,0020 & 0,0009 & 0,0001 & 0,0000 & 0,0000 & 0,0001 & 0,0001 & 0,0011 & 0,0050 & 0,0000 & 0,0020 & 0,0001 & & & & & & & \\
\hline Q4d & 0,4034 & 0,1978 & 0,3262 & 0,1961 & 0,3092 & 0,3556 & 0,4085 & 0,3789 & 0,2429 & 0,1864 & 0,1976 & 0,2295 & 0,3020 & 0,2380 & 0,2440 & 0,7201 & 1,0000 & & & & & \\
\hline & 0,0000 & 0,0039 & 0,0000 & 0,0041 & 0,0000 & 0,0000 & 0,0000 & 0,0000 & 0,0004 & 0,0064 & 0,0039 & 0,0007 & 0,0000 & 0,0005 & 0,0003 & 0,0000 & & & & & & \\
\hline Q5d & 0,3263 & 0,1132 & 0,1746 & 0,1392 & 0,2719 & 0,3259 & 0,3069 & 0,3145 & 0,2413 & 0,2000 & 0,1561 & 0,2170 & 0,3378 & 0,1832 & 0,1884 & 0,5209 & 0,5118 & 1,0000 & & & & \\
\hline & 0,0000 & 0,1019 & 0,0112 & | $0,0429 \mid$ & 0,0001 & 0,0000 & 0,0000 & 0,0000 & 0,0004 & 0,0034 & 0,0233 & 0,0015 & 0,0000 & 0,0075 & 0,0059 & 0,0000 & 0,0000 & & & & & \\
\hline Q6d & 0,0875 & 0,1125 & 0,1863 & 0,1173 & 0,1283 & 0,1341 & 0,1745 & 0,1961 & 0,1830 & 0,0665 & 0,0853 & 0,0716 & 0,1488 & 0,1278 & 0,0667 & 0,1541 & 0,0981 & 0,2386 & 1,0000 & & & \\
\hline & 0,2113 & 0,1048 & 0,0069 & 0,0891 & 0,0628 & 0,0517 & 0,0113 & 0,0042 & 0,0078 & 0,3366 & 0,2185 & 0,3008 & 0,0311 & 0,0639 & 0,3346 & 0,0252 & 0,1555 & 0,0005 & & & & \\
\hline Q9d & 0,0148 & 0,1921 & 0,2235 & 0,1614 & 0,1000 & 0,2920 & 0,3292 & 0,2611 & 0,2500 & 0,2092 & 0,1862 & 0,2254 & 0,2534 & 0,3345 & 0,2571 & 0,3411 & 0,2986 & 0,1842 & 0,3333 & 1,0000 & & \\
\hline & 0,8543 & 0,0150 & 0,0045 & 0,0402 & 0,2057 & 0,0002 & 0,0000 & 0,0008 & 0,0014 & 0,0075 & 0,0177 & 0,0039 & 0,0012 & 0,0000 & 0,0010 & 0,0000 & 0,0001 & 0,0194 & 0,0000 & & & \\
\hline Q10d & 0,2632 & 0,0398 & 0,0613 & 0,1017 & $-0,0117$ & 0,0039 & 0,1274 & 0,0015 & 0,0787 & 0,0360 & $-0,0355$ & 0,0304 & $-0,0448$ & $-0,0774$ & $-0,1357$ & 0,2392 & 0,3131 & 0,1725 & 0,1586 & 0,0369 & 1,0000 & \\
\hline & 0,0059 & 0,6781 & 0,5227 & 0,2838 & 0,9018 & 0,9672 & 0,1806 & 0,9875 & 0,4071 & 0,7048 & 0,7090 & 0,7492 & 0,6387 & 0,4150 & 0,1519 & 0,0107 & 0,0007 & 0,0690 & 0,0965 & 0,7042 & & \\
\hline Q11d & 0,4144 & 0,2383 & 0,2075 & 0,2258 & 0,2391 & 0,2635 & 0,1443 & 0,2540 & 0,2055 & 0,1607 & 0,1395 & 0,0798 & 0,1322 & $-0,0175$ & $-0,0028$ & 0,2731 & 0,2799 & 0,3040 & $-0,0049$ & 0,0717 & 0,4649 & 1,0000 \\
\hline & 0,0000 & 0,0164 & 0,0373 & 0,0218 & 0,0150 & 0,0072 & 0,1480 & 0,0096 & 0,0373 & 0,1049 & 0,1600 & 0,4230 & 0,1855 & 0,8607 & 0,9778 & 0,0053 & 0,0042 & 0,0018 & 0,9609 & 0,4830 & 0,0000 & \\
\hline
\end{tabular}

Fuente: Elaboración propia a partir de los análisis estadísticos. 
La variable que indica el nivel de responsabilidad del profesional contable (Q1d) indica la correlación con la mayoría de las variables, excepto en tres hechos sospechosos de lavado, el conocimiento de la existencia de NOCLAR (Q9d) (poco conocido porque aún no se ha adoptado en Brasil), y la sujeción a inspección (Q6d), que son muy pocos casos de ocurrencia. El último punto, que corresponde al objetivo de la acción de cumplimiento, es una variable que se correlaciona con algunas otras variables, posiblemente, porque pocos profesionales tienen una percepción real de su efectividad.

Con respecto a las variables de reconocimiento de comportamientos sospechosos, todas las variables (Q2) mostraron una correlación significativa entre sí, lo que indica una relación lineal entre ellas, con el mismo nivel de conocimiento. Con respecto al grupo de variables de capacitación y conocimiento (Q3d, Q4d, Q6d, Q9d), el estudio de la legislación de prevención de lavado (Q3d), la norma del consejo profesional (Q4d) y el conocimiento de la existencia de NOCLAR (Q9d), la mayoría de las variables se correlacionaron, lo que indica una relación lineal entre estas variables, a excepción de la variable de inspección y vigilancia (Q6d).

Finalmente, es importante resaltar las variables que indican la propensión a reportar profesionales por canales internos (Q10d) y externos (Q11d). Con respecto a la variable que representa el canal interno (Q10d) (si "sería seguro informar internamente en el defensor del pueblo de su empresa"), los resultados indicaron correlación solo con las variables de responsabilidad (Q1), la legislación de prevención de lavado (Q3d) y el regla del consejo profesional (P4d); el último punto puede indicar el temor a este canal de informes, en el que hay una exposición directa del empleado a los gerentes, mientras que el conocimiento de las reglas podría dar una mayor certeza en cuanto a posibles represalias. En cuanto a la posibilidad de denuncia por canal externo (Q11d) y el organismo de control, los resultados indicaron una correlación positiva y significativa con la responsabilidad (Q1d) y la obligatoriedad (Q5d), con el reconocimiento de varios hechos sospechosos de ilegalidad (Q2), con el estudio de los estándares anti-lavado (Q3, Q4) y con la seguridad del canal interno (Q10d).

El instrumento presentó otro conjunto de afirmaciones para los encuestados para indicar sus percepciones sobre el nivel de responsabilidad y las percepciones de las quejas en diferentes canales de informes. Estas preguntas fueron respondidas bajo las alternativas "Sî" o "No". Los profesionales de contabilidad se identificaron como responsables de llevar a cabo una conducta contra el lavado de dinero. Sin embargo, cabe destacar que solo la mitad de la muestra expresó su opinión sobre la posible denuncia de irregularidades financieras en su empresa independientemente del canal, como se evidencia en la tabla 6.

Tabla 6. Responsabilidad y queja

\begin{tabular}{|c|c|c|c|c|c|c|}
\hline \multirow{2}{*}{ Variable } & \multicolumn{2}{|c|}{ Frecuencia } & \multirow{2}{*}{ N } & \multicolumn{2}{|c|}{ Porcentaje } & \multirow{2}{*}{$\Sigma$} \\
\cline { 2 - 3 } \cline { 5 - 6 } & NO: 0 & SI:1 & & NO: 0 & SÍ: 1 & \\
\hline Q1d & 43 & 165 & 208 & 20,67 & 79,33 & 100,00 \\
\hline Q10d & 13 & 100 & 113 & 11,50 & 88,50 & 100,00 \\
\hline Q11d & 16 & 87 & 103 & 15,53 & 84,47 & 100,00 \\
\hline
\end{tabular}

Fuente: Elaboración propia a partir de los análisis estadísticos.

Los resultados (Q1d) indican que el profesional contable se considera responsable de actuar para combatir el lavado de dinero y los delitos relacionados, con $79,33 \%$ de los encuestados. Es importante resaltar que, como lo destaca la norma (CFC, 2017), el profesional de contabilidad no es un investigador ni sería 
apropiado asignar al profesional con esta asignación adicional. Aun así, el desempeño profesional responsable genera la expectativa de que no ejecute las prácticas ilegales. En una visión estructuralista propuesta por Neu et al. (2013), esto corresponde con el concepto del "contador virtuoso".

Aunque se previó una garantía de anonimato en el instrumento de investigación, el número de respuestas sobre posibles indicaciones de evidencia ilícita alcanzó valores cercanos a la mitad de los encuestados. Los encuestados tuvieron la oportunidad de responder si estarían seguros de informar evidencia de irregularidades financieras que ocurrieron dentro de su empresa (organización del trabajo) interna o externamente con un organismo de control. Del reducido universo de encuestados, $88.5 \%$ y $84.5 \%$ indicaron que tendrían la seguridad para hacerlo.

Como reclamo interno, la pregunta (P10d), además de tener un mayor número de encuestados, llegó a un nivel mayor que el canal externo (P11d) de las agencias de control. Es necesario analizar el resultado de esta cuestión en el contexto, porque un universo significativo de encuestados se ha omitido; además, estos se niegan a asumir una tendencia pro omisión o, incluso, una interpretación divergente de la pregunta propuesta en el instrumento de investigación.

Es interesante presentar en este punto el resultado de la pregunta que señala los "temores" del profesional contable con respecto a la posible denuncia de un acto ilegal inicialmente previsto para la interpretación de las respuestas negativas de denuncia. La pregunta Q12d abordó los factores que mitigan el potencial de presentación de informes del profesional contable. Se propuso la pregunta "Si ha estado al tanto de la evidencia de un acto ilícito, ¿cuál ha sido su mayor temor para informar un hecho que ocurrió en su empresa?”.
El resultado general de las respuestas discursivas permitió identificar la razón principal que puede llevar al profesional contable a no informar una sospecha de irregularidades: ser despedido (la pérdida del empleo como una forma de persecución o represalia, aunque son conceptos más amplios que pueden generar otros "miedos").

El despido o la pérdida del trabajo fue el "miedo" más indicado por los profesionales de la contabilidad, a quienes incluso se aborda. Dicho aspecto es considerado entre los riesgos de persecución/represalia. Además, se indicaron los siguientes casos como posibles resultados de la persecución en el entorno profesional: agresión, conflicto, muerte y castigo. Indirectamente, pero con repercusiones similares, varios señalaron el riesgo de que personas inocentes involucradas sean despedidas e, incluso, que se cierre la empresa con el consecuente despido de empleados.

Otros temores están relacionados a problemas técnicos, como la obtención de datos y documentos de respaldo para demostrar la evidencia, o el riesgo de que esta acción revierta contra el profesional por no probar el hecho. Como resultado de su desempeño profesional, al estar muy cerca de la gestión y el control del flujo financiero, algunos entrevistados señalan el riesgo de ser considerados responsables o involucrados como responsables técnicos o de apoyo. Dicha asunción de responsabilidad podría, incluso, llevarlos a la prisión.

En algunas respuestas, se señaló la posibilidad de frustración por no producir resultados prácticos y perder tiempo, mientras que en otras se comentó el riesgo de exponerse a sus colegas, jefes, subordinados y al mercado. Esto puede afectar la credibilidad profesional de las partes interesadas y provocar una pérdida de reputación. Aunque no están sujetos a despido, los profe- 
sionales independientes señalan riesgos como perder contratos y clientes. De acuerdo con Levitt (2002), dichos riesgos indicarían la existencia de incentivos para ignorar a los clientes ilícitos. En el caso del sector público, la pérdida de la función de confianza se mencionó como un miedo.

\section{Consideraciones finales}

La presente investigación analizó el conocimiento sobre la evidencia del lavado de dinero, y la percepción de los profesionales de la contabilidad con respecto al reconocimiento de su responsabilidad, la seguridad, los medios para informar y los temores con respecto a la efectividad de la comunicación de situaciones con características de tales delitos. Comprender el proceso de racionalización de estas comunicaciones puede hacer que las políticas de gobernanza sean más efectivas a favor de un estado de compliance organizacional; en consecuencia, puede llevar a reducir los riesgos y costos de operación (Júnior \& Moreira, 2011; Gomes et al., 2018).

La clase de contabilidad ha evolucionado hacia el concepto social con la adopción del reglamento de prevención de lavado de dinero desde 2013, bajo la Resolución CFC 1,445/2013. Muchas categorías profesionales no implementan estándares rectores para sus regulados. Aun así, la pequeña cantidad de comunicaciones para el organismo de control (CFC, 2019; COAF, 2019) es lo suficientemente preocupante para motivar la implementación de esta investigación.

Se analizaron y destacaron los puntos considerados relevantes para el comportamiento del profesional. El profesional contable se considera responsable de actuar para combatir el lavado de dinero, como se evidencia en el 79,33\% de los encuestados. Aunque no busca hechos, se espera que el profesional responsable no realice conductas ilícitas, incluida la identificación y comunicación de aquellos percibidos dentro del alcance de su actividad laboral (CFC, 2017).

El nivel de capacitación sobre el tema no es alto, lo que se evidenció en que $60 \%$ de los encuestados respondieron afirmativamente a este punto. En parte, eso puede ser una limitación debido a las reglas de capacitación continúas proporcionadas para los profesionales de la contabilidad que alcanzan solo unos pocos segmentos, como auditores, peritos y gerentes técnicos. Este factor es relevante porque es necesario comprender el estándar para poder cumplirlo. Es aún más preocupante cuando se habla de la incorporación de la norma internacional NOCLAR.

A pesar del bajo nivel de capacitación indicado, los encuestados indicaron las diversas características de los signos de lavado de dinero con un conocimiento promedio de 6,95. Algunas de estas circunstancias con menor conocimiento y mayor desviación estándar pueden hacer que el proceso de comunicación sea inocuo. En estos casos, las operaciones de lavado de dinero podrían estar ocurriendo sin que el profesional contable sea capaz de darse cuenta de este hecho, lo que abre la posibilidad a que los sistemas computarizados realicen búsquedas automatizadas en bases de datos para identificar parámetros preestablecidos.

A pesar del anonimato indicado, los encuestados tienen miedo de responder sobre la posibilidad de comunicación sobre un hecho que ocurrió en su organización. Solo la mitad de los encuestados opinó. Los resultados indican que los profesionales contables están seguros de reportar evidencia de ilícitos financieros de su compañía internamente y externamente (con $88,5 \%$ y $84,5 \%$ respectivamente), lo que se corrobora con estudios que señalan una mayor

Contabilidad y Negocios (16) 31, 2021 / e-ISSN 2221-724X 
propensión a quejas a través del canal interno de la organización (Park et al., 2009). Aun así, estos altos porcentajes de propensión a reportar comportamientos ilícitos pueden no representar comportamientos, porque los efectos de la respuesta "no comunicar" tiene el mismo efecto de no responder a la cuestión. Este caso fue frecuente.

El análisis de correlación mostró la existencia de una relación lineal entre algunas variables. Entre las variables de conocimiento sobre circunstancias de actividad ilícita, la relación percibida fue lineal entre todas las variables. Se demostró que la percepción de responsabilidad está relacionada con el predominio de variables, así como la percepción de seguridad para la comunicación por canales externos, similar a la de la UIF. Este no es el caso con la comunicación por canal interno, que se correlacionaba con pocas variables. Este análisis de correlación entre variables introduce la posibilidad de aplicar modelos inferenciales, cuyas construcciones se pueden relacionar con variables no correlacionadas, como la percepción del conocimiento y la capacitación, así como la percepción de seguridad.

Los factores atenuantes minimizan la posibilidad de comunicación de tales circunstancias; estas fueron tratadas en la investigación como los "miedos" del profesional, que fueron analizados y discutidos. El principal temor percibido fue la pérdida de trabajo debido a represalias o acoso, sin perjuicio de otros riesgos percibidos que van desde la integridad hasta la reputación. También existe un temor técnico, como en el caso de pruebas y documentación de respaldo. Este podría ser el tema de un marco legal más claro sobre la responsabilidad de informar, así como los actos y hechos técnicos contables. Aun así, la regla de categoría profesional enfatiza que no hay responsabilidad civil derivada de la comunicación de buena fe, además de resaltar la confidencialidad de la comunicación (CFC, 2017).

\section{Referencias bibliográficas}

Albrecht, C.; Kranacher, M., \& Albrecht, S. (2008). Asset Misappropriation White Paper for the Institute for Fraud Prevention. Recuperado de http://www.theifp. org/research-grants/IFP-Whitepaper-5.pdf [Consulta: 30 de octubre de 2018].

Alldridge, P. (2008). Money Laundering and Globalization. Journal of Law and Society, 35(4), 437-463. https:// doi.org/10.1111/j.1467-6478.2008.00446.x

Amorim, E. N. C., Cardozo, M. A., \& Vicente, E. F. (2012). Os impactos da implementação de controles internos, auditoria e compliance no combate e prevençáo à lavagem de dinheiro no Brasil. Enfoque: Reflexão Contábil, 31(3), 23-35. https://doi.org/10.4025/enfoque. v31i3.15616

Apostolou, B., Apostolou, N., \& Thibadoux, G. (2015). Horseplay in Dixon: Lessons Learned from the Rita Crundwell Fraud. Journal of Forensic \& Investigative Accounting, 7(1), 275-289.

Association of Certified Fraud Examiners (ACFE). (2018). Report to the Nations. 2018 Global Study on Occupational Fraud and Abuse. Recuperado de https:// s3-us-west-2.amazonaws.com/acfepublic/2018-reportto-the-nations.pdf [Consulta: 12 de febrero de 2019].

Campos, F. A. O., \& Pereira, R. A. (2016). Corrupção e ineficiência no Brasil: Uma análise de equilíbrio geral. Estudos Econômicos, 46(2), 373-408. https://doi. org/10.1590/0101-416146244rpf

Coates, J. C. IV. (2007). The Goals and Promise of the Sarbanes-Oxley Act. Journal of Economic Perspectives, 21(1), 91-116. https://doi.org/10.1257/jep.21.1.91

Coelho Junior, F. A., \& Abbad, G. (2010). Construção e validaçấo de uma escala de avaliação de impacto em profundidade de um treinamento a distância em uma organização do setor bancário brasileiro. Revista Eletrônica de Administraçâo - Read, 16(1), 1-28.

Conselho de Controle de Atividades Financeiras (COAF). (2018). Supervisão. Brasilia: COAF. Recuperado de 
http://www.fazenda.gov.br/orgaos/coaf [Consulta: 10 de mayo de 2018].

Conselho de Controle de Atividades Financeiras (COAF). (2019). Coafem números. Brasilia: COAF. Recuperado de https://www.fazenda.gov.br/orgaos/coaf/coaf\#coafem-numeros [Consulta: 30 de abril de 2019].

Conselho Federal de Contabilidade (CFC). (2013, 26 de julio). Dispóe sobre os procedimentos a serem observados pelos profissionais e Organizaçóes Contábeis, quando no exercício de suas funçóes, para cumprimento das obrigaçóes previstas na Lei No 9.613/1998 e alteraçôes posteriores [Resolução CFC No 1.445]. Brasilia: Governo Federal.

Conselho Federal de Contabilidade (CFC). (2017, 22 de setiembre). Dispóe sobre os procedimentos a serem observados pelos profissionais e Organizaçóes Contábeis, quando no exercício de suas funçôes, para cumprimento das obrigaçóes previstas na Lei $\mathrm{N}^{\circ}$ 9.613/1998 e alteraçóes posteriores [Resoluçáo CFC No 1.530]. Brasilia: Governo Federal.

Conselho Federal de Contabilidade (CFC). (2019). Quantos Somos. Recuperado de https://cfc.org.br/registro/ quantos-somos-2/ [Consulta: 20 de abril de 2019].

Cronbach, L. J. (1951). Coefficient Alpha and the Internal Structure of Tests. Psychometrika, 16(3), 297-334. https://doi.org/10.1007/BF02310555

Da Silva, J., Marques, L., \& Teixeira, R. (2011). Prevenção à lavagem de dinheiro em instituições financeiras: avaliação do grau de aderência aos controles internos. BASE-Revista de Administração e Contabilidade da Unisinos, 8(4), 300-310. https://doi.org/10.4013/base.2011.84.03

Fávero, L. P., Belfiore, P., Takamatsu, R., \& Suzart, J. (2014). Métodos Quantitativos com Stata: Procedimentos, Rotinas e Análise de Resultados (Vol. 1). Río de Janeiro: Brasil.

Gomes, H. O., Ramos, M. O., Silva, M. V. D., \& Santos, L. M. V. D. (2018). A contabilidade do crime no Brasil: Avanços e desafios. Revista Evidenciação Contábil
\& Finanças, 6(2), 81-94. https://doi.org/10.18405/ RECFIN20180205

Grandis, R. (2011): O exercício da advocacia e o crime de lavagem de dinheiro. En C. V. De Carli (Coord.), Lavagem de dinheiro: prevenção e controle penal (pp. 115-146). Porto -Alegre: Verbo Jurídico.

Gupta, S., Davoodi, H., \& Tiongson, E. (2001). Corruption and the Provision of Health Care and Education Services. En A. K. Jain (Ed.), The Political Economy of Corruption (pp. 111-141). New York: Routledge.

Gupta, S., Sharan, R., \& Mello, L. (2000). Corruption and Military Spending (IMF Working Paper 00/23). Washington, D. C.: Fondo Monetario Internacional. https://doi.org/10.5089/9781451844030.001

Jatahy, P. J. C., \& Vieira, M. M. F. (2004). Mudança organizacional, controle e desempenho: a experiência do Banco Central do Brasil com o combate à lavagem de dinheiro. Revista Organizaçôes \& Sociedade: O\&S, 11(31), 77-101. https://doi.org/10.1590/S1984-92302004000300005

Jung, L. W. (2007). Lavagem de dinheiro e a responsabilidade do contador. Revista Catarinense da Ciência Contábil, 6, 39-54. https://doi.org/10.16930/2237-7662/ rccc.v6n17p39-54

Júnior, I. J. N., Moreira, E. M. S. (2011). Perícia contábil: uma ferramenta de combate ao crime organizado [Edición especial]. Revista de Educação e Pesquisa em Contabilidade, 5, 126-153. https://doi.org/10.17524/ repec.v5i0.169

Kriekebaum, H. (2008). Corruption as a Moral Issue. Social Responsibility Journal, 4(1/2), 82-88. https:// doi.org/10.1108/17471110810856857

Lehman, C. R., \& Okcabol, F. (2005). Accounting for Crime. Critical Perspectives on Accounting, 16, 613-639. https://doi.org/10.1016/j.cpa.2003.08.003

Levitt, S. D. (2002). Using Electoral Cycles in Police Hiring to Estimate the Effects of Police on Crime: Reply. American Economic Review, 92(4), 1244-1250. https://doi.org/10.1257/00028280260344777

Contabilidad y Negocios (16) 31, 2021 / e-ISSN 2221-724X 
Martins, P. S. (2011). Lavagem de dinheiro transnacional e obrigatoriedade da ação penal. Belo Horizonte: Arraes Editores.

Mauro, P. (1995). Corruption and Growth. Quarterly Journal of Economics, 110(3), 681-712. https:/doi. org/10.2307/2946696

Moro, S. F. (2010). Crime de lavagem de dinheiro. São Paulo: Saraiva.

Neu, D., Everett, J., Rahaman A.S., \& Martinez, D. (2013). Accounting and Networks of Corruption Accounting. Organizations and Society, 38(6), 505-524. https://doi. org/10.1016/j.aos.2012.01.003

Neves Júnior, I. J., \& Moreira, E. M. de S. (2011). Perícia contábil: uma ferramenta de combate ao crime organizado [Edición especial]. Revista de Educação e Pesquisa em Contabilidade (REPeC), 5, 126-153. https://doi. org/10.17524/repec.v5i0.169

Pacini, C. J., Hopwood, W. S., \& Sinclair, D. T. (2016). Domestic Asset Tracing: Identifying, Locating and Freezing Stolen and Hidden Assets. Journal of Forensic Accounting Research, 1(1), A42-A65. https://doi. org/10.2308/jfar-51549

Presidência da República. (1998, 3 de marzo). Lei que dispóe sobre os crimes de "lavagem" ou ocultação de bens, direitos e valores; a prevenção da utilização do sistema financeiro para os ilícitos previstos nesta Lei; cria o Conselho de Controle de Atividades Financeiras - COAF, e dá outras providências [Lei N 9.613]. Brasilia: Governo Federal.

Presidência da República. (2012, 9 de julio). Lei que altera a Lei n. 9.613, de 3 de março de 1998, para tornar mais eficiente a persecução Penal dos crimes de lavagem de dinheiro [Lei $N^{\circ}$ 12.683]. Brasilia: Governo Federal.

Ramos, P. R. (2010). Corrupção na Administração Pública e crimes de "lavagem" ou ocultação de bens, direitos e valores. Revista Mineira de Contabilidade, 4(40), 14-22.
Receita Federal do Brasil (RFB). (2017). Receita Federal Regulamenta a obrigatoriedade. Recuperado de http:// www.fazenda.gov.br/noticias/2017/novembro/receitafederal-regulamenta-a-obrigatoriedade-de-prestacaode-informacoes-em-operacoes-liquidadas-em-especie [Consulta: 30 de abril de 2018].

Ribeiro, A. A. D., Rodrigues, R. N., Prazeres, R. V., \& Araújo, J. G. (2016). Um estudo sobre a relevância da contabilidade forense como instrumento de investigação: A percepção de profissionais ligados ao combate à lavagem de capitais. Revista de Gestão, Finanças e Contabilidade, 6(1), 45-75. https://doi. org/10.18028/2238-5320/rgfc.v6n1p45-75

Silva, J. L. R., Marques, L. F. B., \& Teixeira, R. (2011). Prevenção à lavagem de dinheiro em instituiçôes financeiras: avaliação do grau de aderência aos controles internos. BASE - Revista de Administração e Contabilidade da UNISINOS, 8(4), 300-310. https://doi.org/ $10.4013 /$ base. 2011.84 .03

Simon, C. A. (2012). Individual Auditors' Identification of Relevant Fraud Schemes. Auditing: A Journal of Practice \& Theory, 31(1), 1-16. https://doi.org/10.2308/ ajpt-10169

Svensson, J. (2003). Who Must Pay Bribes and How Much? Evidence from a Cross Section of Firms. The Quarterly Journal of Economics, 118(1), 207-230. https://doi. org/10.1162/00335530360535180

Wechsler, W. (2001). Follow the Money. Foreign Affairs. Recuperado de https://www.foreignaffairs.com/articles/2001-07-01/follow-money [Consulta: 12 de mayo de 2018].

Fecha de recepción: 04 de diciembre de 2019

Fecha de aceptación: 08 de junio de 2020 Correspondencia: jonatas.sallaberry@hotmail.com leonardo.flach@gmail.com 